\title{
Energy Conservation in IEEE 802.15.4 Compliant Wireless Sensor Network using LT codes
}

\author{
V. Nithya \\ Department of Electronics and \\ Communication Engineering, \\ SRM University, \\ Chennai, India.
}

\author{
B. Ramachandran \\ Department of Electronics and \\ Communication Engineering, \\ SRM University, \\ Chennai, India.
}

\author{
K. Muruganand \\ Department of Electronics and \\ Communication Engineering, \\ SRM University, \\ Chennai, India.
}

\begin{abstract}
In this paper, we investigate the energy efficiency of a IEEE 802. 15. 4 based RF transceiver using Direct Sequence Spread Spectrum- Offset Quadrature Phase Shift Keying (DSSSOQPSK) modulation under $2.4 \mathrm{GHz}$ frequency band in a WSN. Multi path propagation in a wireless environment affects the reliability of the network and therefore the sensor nodes wastes the battery power to ensure successful delivery of data by adopting Automatic Repeat reQuest (ARQ) schemes. Error control coding is one of the most suitable solutions to this reliability issue. In this paper, the use of one such Forward Error Correction (FEC) codes such as Luby Transform (LT) codes to reduce energy expenditure by the network nodes is analyzed under Rayleigh fading channel environment. The performance metrics considered for our study are Bit Error Rate (BER) and Energy spent per bit. Simulation results show that LT codes show improved performance under severe fading channel conditions, i. e., for low SNR values ( -5 to $2 \mathrm{~dB}$ ) and ARQ scheme provides better performance under high SNR values (above $2 \mathrm{~dB}$ ).
\end{abstract}

\section{Keywords}

Forward Error Correction, IEEE 802. 15. 4, LT codes, Reliability, Wireless Sensor Network, Zigbee.

\section{INTRODUCTION}

Wireless sensor networks (WSNs) have received a lot of attention in the recent years. WSNs are typically composed of a large number of sensor nodes that are very tiny, low cost, multi-functional and have limited energy resource. Since the applications that involve wireless sensor networks require long system lifetimes, energy must be used efficiently [1]. Moreover, the application can fulfill its requirement if the communication through the network is reliable. This is because, the nature of the communication channel used for sensor data transmission is erroneous due to the presence of thermal noise, multiple interferers in the same network, interference from outside the network, large scale and small scale fading components due to multipath propagations. The reliability of the communication link is generally studied in terms of Symbol Error Rate (SER). This greatly affects network performance in terms of throughput, delay and energy [2].

The optimum packet size based on energy efficiency over frequency non-selective Rayleigh fading channel is derived [3]. The energy efficiency of error control schemes for wireless sensor networks is evaluated for a Nakagami-m fading channel [4]. Similarly, the authors [5] have analyzed error performance and capacity of Low Density Parity Check (LDPC) codes for Nakagami-m fading channels. Further work [6] discusses an optimum Forward Error Correction (FEC) scheme with maximum energy efficiency in a sensor network.
The comparison of energy performance of various packet delivery schemes is made in the presence of Rayleigh fading channels [7]. The performance of Repeat- Accumulate codes in single hop WSN over Convolutional codes is studied [8].

It is observed that most of the earlier work uses FECs to achieve reliable communication thereby reducing the channel bit error rate and maximize the throughput. They have not considered FECs for energy efficiency analysis [9]. In this work, we consider a IEEE 802.15.4 compliant sensor network for our study. Henceforth, in this paper a wireless sensor node based on Zigbee technology using IEEE 802.15.4 RF transceiver will be referred to as IEEE 802.15.4 Zigbee transceiver.

The main contribution of this paper can be twofold: They are (i) BER analysis of IEEE 802.15.4 Zigbee transceiver using LT codes, and (ii) Energy expenditure analysis of the network using LT coded IEEE 802.15.4 Zigbee RF transceiver over Rayleigh fading channels.

The rest of the paper is organized as follows: The LT Coded IEEE 802.15.4 Zigbee RF transceiver model for WSN is detailed in Section II. Section III describes the simulation model. Results and discussions are presented in Section IV. Finally, Section V presents the conclusions.

\section{LT CODED IEEE 802. 15. 4 RF TRANSCEIVER FOR WSN}

We consider a wireless sensor network consisting of sensor nodes that use IEEE 802.15.4 Zigbee transceiver under 2.4 GHz frequency band, e.g., TelosB, MicaZ. The IEEE 802.15.4 standard has been adopted by Zigbee for WSN technology. The salient features of Zigbee include low cost, very low power consumption, reliable data transfer and ease of implementation.

\subsection{IEEE 802.15.4 PHY Layer}

The PHY layer of IEEE 802.15.4 standard can be operated in three unlicensed frequency bands namely $858 \mathrm{MHz}, 915 \mathrm{MHz}$ and $2.4 \mathrm{GHz}$. Accordingly, the standard specifies three different physical media: (i) Direct Sequence Spread 


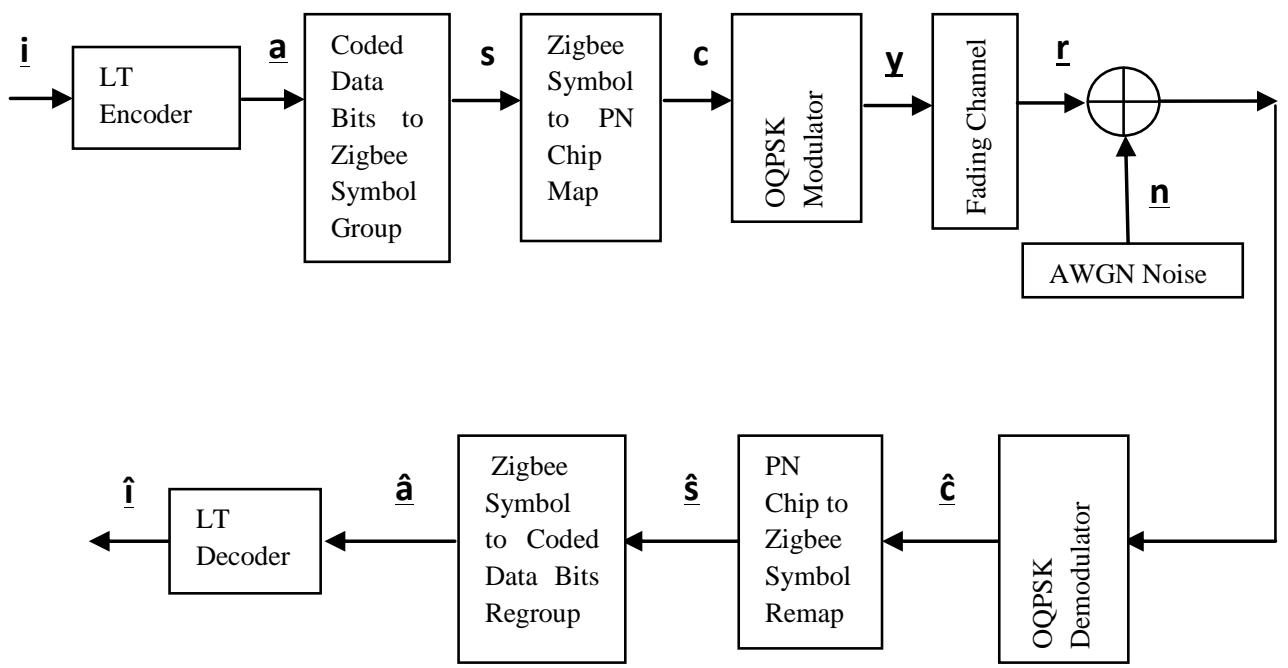

Figure 1. Block Diagram of LT Coded Zigbee IEEE 802.15.4 RF Transceiver

Spectrum using BPSK operating in the frequency range of $868 \mathrm{MHz}$ at a data rate of $20 \mathrm{Kbps}$, (ii) Direct Sequence Spread Spectrum using BPSK operating in the frequency band of $915 \mathrm{MHz}$ at a data rate of $40 \mathrm{Kbps}$, (iii) Direct Sequence Spread Spectrum using O-QPSK operating in the frequency band of $2.4 \mathrm{GHz}$ at a data rate of $250 \mathrm{Kbps}$. For analysis purposes in this work, we have considered higher data rate physical media (2.4 GHz/250 Kbps) which is an internationally used license free ISM frequency band.

The specifications of IEEE 802.15.4 Zigbee transceiver operated with $2.4 \mathrm{GHz}$ band is as follows: The data modulation scheme used here is Direct Sequence Spread Spectrum-Offset Quadrature Phase Shift Keying (DSSSOQPSK). The complete block diagram of Coded IEEE 802.15.4 Zigbee RF transceiver system is shown in Figure 1. This is our proposed modified IEEE 802. 15. 4 Zigbee RF transceiver block diagram, where FEC encoder and decoder blocks are included in conventional functional block diagram. It involves spreading and modulation of input bits. In the first stage, incoming bits are grouped into four, so as to represent a Zigbee symbol. These four bits are used to select one of the 16 nearly orthogonal Pseudo random Noise (PN) sequences to be transmitted. The mapping of symbols to chips is achieved through 32-chip PN sequences shown in Table I. The PN sequences are related to each other through cyclic shifts and the successive selected PN sequences are concatenated and sent to the OQPSK modulator.

The incoming chip sequences to the OQPSK modulator are modulated onto the carrier with half-sine pulse shaping. The half-sine pulse shaping [10] used to represent each baseband chip is given by

$$
p(t)=\left\{\begin{array}{l}
\sin \left(\frac{\pi t}{2 T_{c}}\right), 0 \leq t \leq 2 T_{c} . \\
0, \text { otherwise }
\end{array}\right.
$$

The modulated signal transmitted through the channel gets affected by white noise and other fading parameters depending upon the channel conditions. In this paper, we have considered Rayleigh fading channels to study network performance over severe fading situations. The receiver section of Zigbee consists of the blocks to perform reverse operations as that of the transmitter. This includes demodulation, chip to Zigbee symbol remapping, and finally Zigbee symbol to regrouping followed by decoding.

\subsection{Luby Transform}

In this paper we have used Luby Transform (LT) codes to achieve forward error correction and thereby aim to improve network lifetime. LT is first practical implementation of Fountain codes $[11,12]$. Any number of encoding symbols $\mathrm{N}$ can be independently generated from $\mathrm{K}$ information symbols by the following encoding process. Fountain codes are from class of codes called rate less codes. Any number of encoding symbols can be independently generated from $\mathrm{K}$ information symbols by the following encoding process as shown in the Figure 2:

1) Determine the degree $d$ of an encoding symbol. The degree is chosen at random from a given node degree distribution $\mathrm{P}(\mathrm{x})$.

2) Choose $d$ distinct information symbols uniformly at random. They will be neighbours of the encoding symbol.

3) Assign the XOR of the chosen d information symbols to the encoding symbol.

The design of LT codes is mainly influenced by the distribution function; Distribution function helps in choosing the combination of information symbols that are to be Xored. Successful decoding also depends on the distribution function $[11,12]$. The basic property required of a good degree Distribution is that input symbols are added to the ripple at the same rate as they are processed. This property is the inspiration for the name Soliton distribution, as a soliton wave is one where dispersion balances refraction perfectly.

For decoding of LT codes, a decoder needs to know the neighbours of each encoding symbol as depicted in Figure 3. This information can be transferred in several ways. 
Table 1. Zigbee Symbol to Chip Mapping [8]

\begin{tabular}{|c|c|}
\hline Zigbee Symbol & $\begin{array}{c}\text { Chip Values } \\
\left(c_{0} c_{1} \ldots c_{30} c_{31}\right)\end{array}$ \\
\hline 0000 & 11011001110000110101001000101110 \\
\hline 1000 & 11101101100111000011010100100010 \\
\hline 0100 & 00101110110110011100001101010010 \\
\hline 1100 & 00100010111011011001110000110101 \\
\hline 0010 & 01010010001011101101100111000011 \\
\hline 1010 & 00110101001000101110110110011100 \\
\hline 0110 & 11000011010100100010111011011001 \\
\hline 1110 & 10011100001101010010001011101101 \\
\hline 0001 & 10001100100101100000011101111011 \\
\hline 1001 & 10111000110010010110000001110111 \\
\hline 0101 & 01111011100011001001011000000111 \\
\hline 1101 & 01110111101110001100100101100000 \\
\hline 0011 & 00000111011110111000110010010110 \\
\hline 1011 & 01100000011101111011100011001001 \\
\hline 0111 & 10010110000001110111101110001100 \\
\hline 1111 & 11001001011000000111011110111000 \\
\hline
\end{tabular}

For example, a transmitter can send a packet, which consists of an encoding symbol and the list of its neighbours. An alternative method is that the encoder and the decoder share a random number generator seed, and the decoder finds out the neighbours of each encoding symbol by generating random linear combinations synchronized with the encoder. With the encoding symbols and the indices of their neighbours, the decoder can recover information symbols with the following three-step process

1) (Release) All encoding symbols of degree one, i.e., those which are connected to one information symbols, are released to cover their unique neighbour.

2) (Cover) The released encoding symbols cover their unique neighbour information symbols. In this step, the covered but not processed input symbols are sent to ripple, which is a set of covered unprocessed information symbols gathered through the previous iterations.

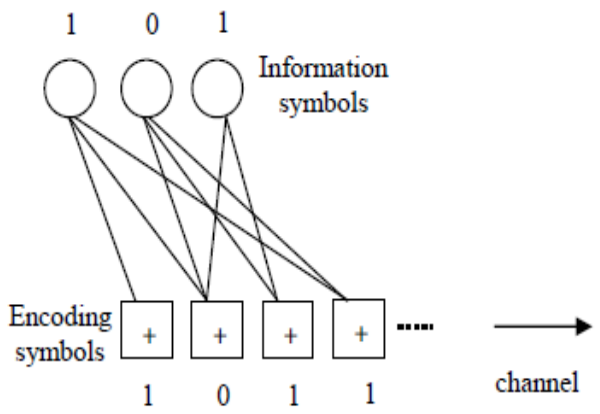

Figure 2. LT Encoder Structure [13]
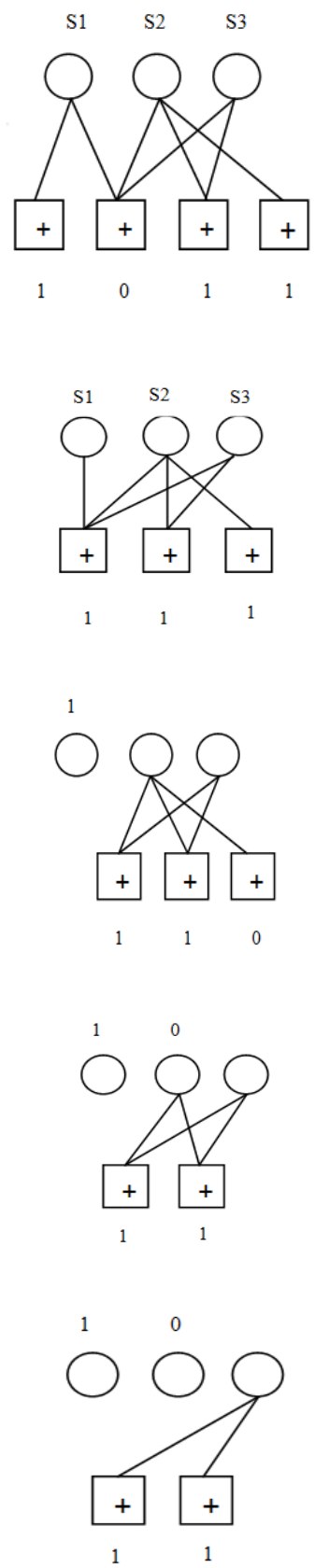

Figure 3. LT Decoder Structure 
3) (Process) One information symbol in the ripple is chosen to be processed the edges connecting the information symbol to its neighbour encoding symbols are removed and the value of each encoding symbol changes according to the information symbol. The processed information symbol is removed from the ripple.

\section{SIMULATION MODEL}

The step-wise procedure for simulating LT coded IEEE 802.15.4 Zigbee transceiver (Figure 1) in MATLAB is given below:

1. First, the information bits $\underline{\mathbf{i}}$ to be transmitted are given to a LT encoder to generate coded bits $\underline{\mathbf{a}}$.

2. Every four bits of binary data stream generated is grouped to form a Zigbee Symbol $\underline{\mathbf{s}}$.

3. Each of these 16 Zigbee symbol $\underline{\mathbf{s}}$ is mapped to the 32-chip $\mathbf{c}$ PN sequence as shown in Table 1 .

4. The chip sequence is then sent as an input to the OQPSK modulator where half-sine pulse shaping of the incoming chips is performed.

5. The modulated signal $\mathbf{y}$ is later transmitted through a wireless channel where the channel noise $\mathbf{n}$ is being added to the transmitted signal. The coefficients of Rayleigh fading channel are generated from two independent Gaussian random variables with mean zero and variance $\sigma_{n}^{2}$.

6. The decision about the transmitted signal from the received signal $\mathbf{r}$ is made by computing the minimum of the Euclidean distance between the received and the reference signal.

7. The estimate of the transmitted symbols $\underline{\hat{\mathbf{s}}}$ is obtained

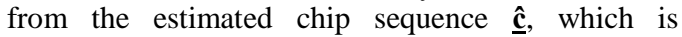
available at the demodulator output.

8. The reverse process of chip to Zigbee symbol remapping and symbol to bits regrouping are done.

9. The bit error rate is obtained by dividing the bit error count by the total number of information bits transmitted.

10. The energy efficiency of the wireless sensor network can be calculated in terms of energy spent per bit. The parameters used in the calculation are based on CC2420 IEEE 802.15.4 Zigbee transceiver chip and Texas Instrument's MSP 430 Microcontroller with $10 \mathrm{~KB}[8]$.

The network parameters used in our simulation are taken from the IEEE 802.15.4 standard values as specified below in the Table II.

\section{RESULTS AND DISCUSSIONS}

The simulations of WSN for the specified network parameters are performed and the performance of Zigbee transceiver in terms of BER and Energy spent per bit is studied as a function of the transmitted SNR. The fading channel is modeled as a Finite State Markov Chain (FSMC) and the total number of FSMC states considered for simulation is 10 . In a slow fading environment, the channel transits to its immediate next state or immediate previous state or stays in the same state. But we have assumed that the channel transits to its immediate next state and the channel
Table 2. Network Parameters [8]

\begin{tabular}{|c|l|}
\hline Parameter & Values \\
\hline$I_{t}(\mathrm{~mA})$ & 21 \\
\hline$I_{r}(\mathrm{~mA})$ & 23 \\
\hline$V_{r}(\mathrm{v})$ & 3.6 \\
\hline$l$ & 256 \\
\hline$R_{b}(\mathrm{kbps})$ & 250 \\
\hline Overhead H+FCS (bytes) & 11 \\
\hline
\end{tabular}

stays in a same state for a period of 100 bits. Here, the error control schemes considered for simulation are ARQ, which is an optional reliability mechanism available in IEEE 802.15.4 Zigbee RF transceiver with maximum number of retries as 7 and default value is set to as 3 and LT codes for different values of $\mathrm{K}$ and $\mathrm{N}$.

Figure 2 shows the BER analysis of LT codes for a given K value and different $\mathrm{N}$ values. The two sample values of $\mathrm{N}$ considered for our study are $\mathrm{N}=856$ and 204 with fixed $\mathrm{K}$ as 104. It is observed that as the number of redundant bits generated are more for $\mathrm{N}=856$ case, the rate of error correction is also more compared to that of $\mathrm{N}=204$ case where the redundancy is comparatively less. This basic underlying principle decides the quality of the service that can be achieved by the LT codes. The energy spent per bit analysis depicted in figure 3 shows that the energy consumed is more for the FEC of high redundancy, i.e., $\mathrm{N}=856$ in comparison with $\mathrm{N}=204$.

Figure 4 and 5 illustrates the performance of LT codes in another dimension. Here, we have fixed the generated encoded symbols $\mathrm{N}$ and varied the information symbols size, $\mathrm{K}$, as 104, 204 and 404. It is found that BER performance of LT codes of various $\mathrm{K}$ values remains almost the same for low SNR range, i.e. , from $-5 \mathrm{~dB}$ to $0 \mathrm{~dB}$, whereas the curves shifts downwards for $\mathrm{K}=104$, under good channel conditions for SNRs above $0 \mathrm{~dB}$. Similarly, the energy spent per bit is found to be higher for LT code with higher redundancy $(\mathrm{K}=104)$.

From the above discussions, it is suggested to choose LT code with $\mathrm{K}=204$ and $\mathrm{N}=856$ as it provides a better tradeoff between energy and error performance when compared to all other code options. Now, we compare the coded system performance over an uncoded network's performance as shown in the figures 6 and 7. ARQ scheme which is available as an option in IEEE 802.15.4 standard is also considered here for study. It is clear that the LT code offers better error performance under severe fading channel conditions when compared to ARQ scheme. There is a cross over in the graph at $\mathrm{SNR}=2 \mathrm{~dB}$, above which ARQ mechanism shows reduced BER. Similar kind of performance is also observed in figure 7 where, the energy spent per bit is found to be very low for lower SNR ranges i.e., from SNR $=-5$ to $2 \mathrm{~dB}$. However, the ARQ scheme outperforms the LT code for SNR values above $2 \mathrm{~dB}$ as in the case of BER. 


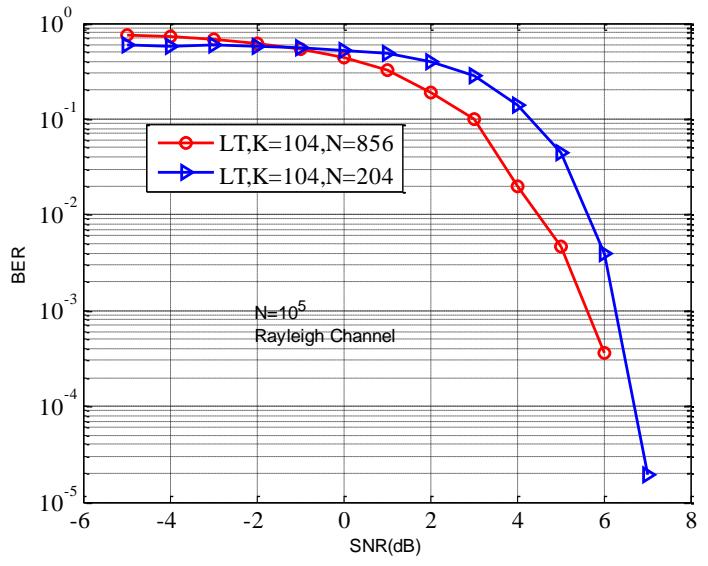

Figure 4. BER analysis of IEEE 802. 15. 4 Zigbee transceiver using $L T$ codes having same $K$ and different $N$.

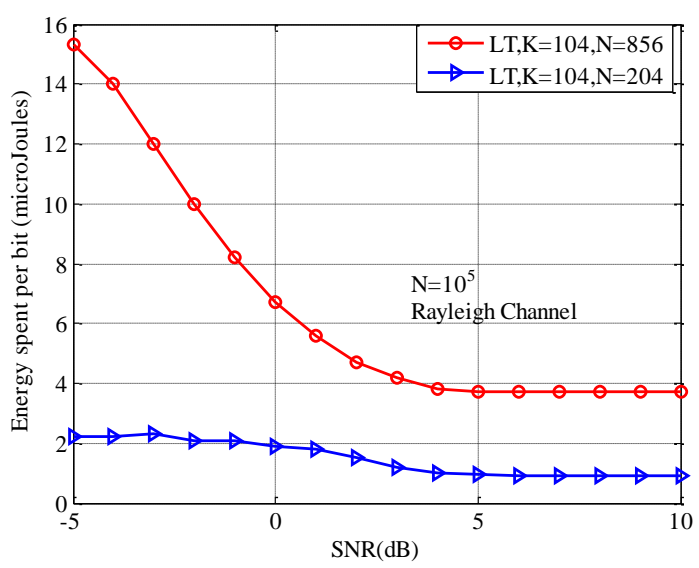

Figure 5. Energy spent per bit by IEEE 802. 15. 4 Zigbee transceiver using $\mathrm{LT}$ codes having same $\mathrm{K}$ and different $\mathrm{N}$.

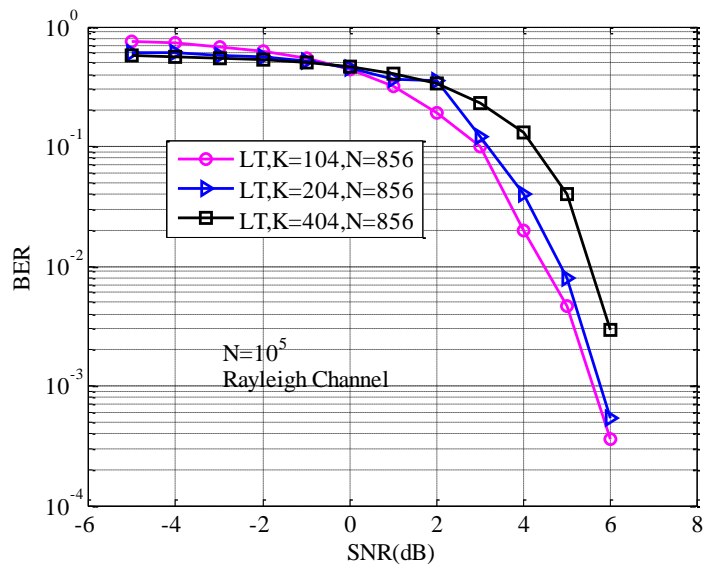

Figure 6. BER analysis of IEEE 802. 15. 4 Zigbee transceiver using $L T$ codes having same $N$ and different $K$.

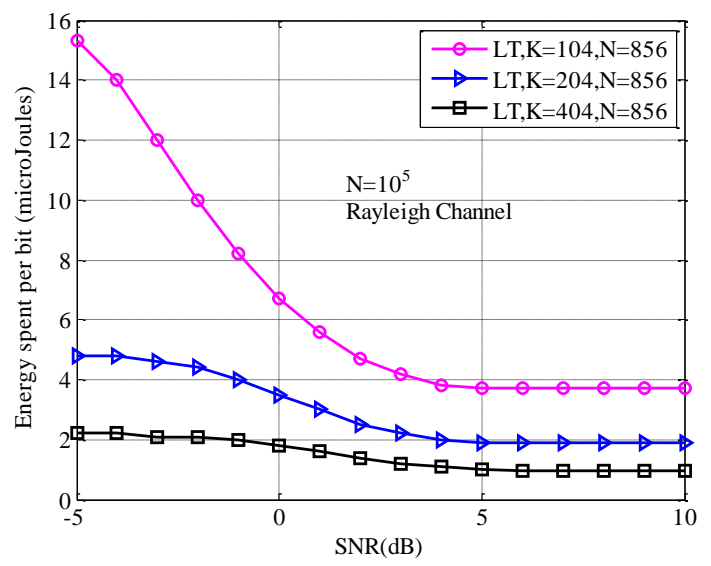

Figure. 7 Energy Spent per bit by IEEE 802.15. 4 Zigbee transceiver using $L T$ codes having same $N$ and different $K$.

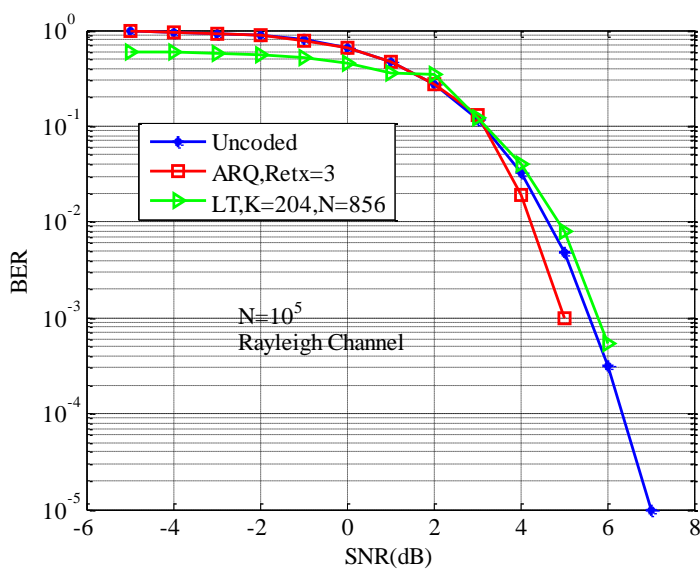

Figure 8. Comparison of BER of IEEE 802. 15. 4 Zigbee transceiver using $L T$ codes over ARQ scheme.

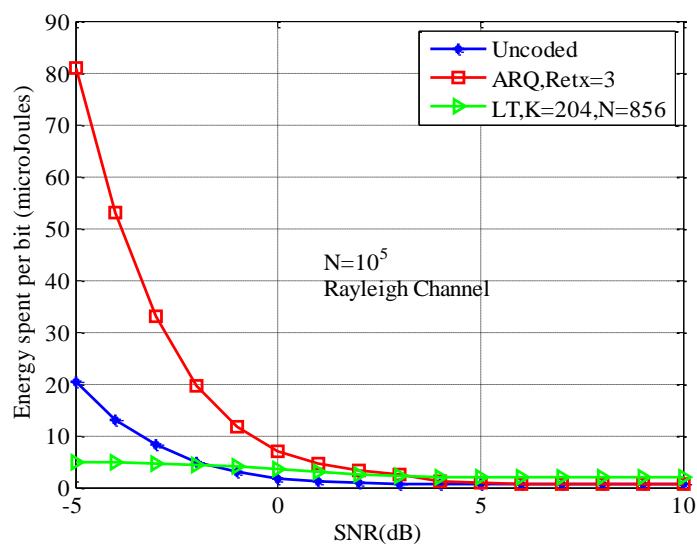

Figure 9. Comparison of Energy Spent per bit of IEEE 802 15. 4 Zigbee transceiver using LT codes over ARQ scheme. 


\section{CONCLUSIONS}

Energy conservation is a critical challenge in resource constrained WSN. The use of FECs to improve the network lifetime of IEEE 802.15.4 compliant sensor network is proposed in this work. For this purpose, LT codes, a class of rate less codes (also called fountain codes) are considered for this study. The ARQ scheme, default error correcting mechanism provided by IEEE 802.15.4 standard is also analyzed. The simulation results shows that use of LT codes in IEEE 802.15.4 Zigbee RF transceiver improves the error and energy performance under worse channel conditions whereas ARQ scheme outperforms well under high SNR values. The incorporation of other variants of fountain codes such as Raptor codes with different degree distribution function to improve the network performance still further can be considered as a future work

\section{REFERENCES}

[1] I. F. Akyildiz, W. Su, Y. Sankarasubramaniam, and E. Cayirci, "A Survey on Sensor Networks" IEEE Communications Magazine, vol. 40, no. 8, pp. 102-114, Aug. 2002.

[2] K. Romer, and F. Mattern, "The design space of wireless sensor networks," IEEE Wireless Communications, vol. 11, no. 6, pp. $54-61$, Dec. 2004.

[3] Y. Sankarasubramaniam, I. F. Akyildiz, and S. W. Mclaughlin, "Energy efficiency based packet size optimization in wireless sensor networks," Proceedings of the First IEEE International Workshop on Sensor Network Protocols an Applications 2003, Alaska, USA, pp. 1-8, May 2003.

[4] J. H. Kleinschmidt, W. C. Borelli, and M. E. Pellenz, "An Analytical Model for Energy Efficiency of error control schemes in Sensor Networks," IEEE International Conference on Communications, Glasgow, Scotland, pp. 3895-3900, 24 - 28, June 2007.

[5] M. R. Islam and J. Kim, "Capacity and BER analysis for Nakagami-m channel in LDPC coded wireless sensor network," International Conference on Intelligent
Sensors, Sensor Networks, and Information Processing, Sydney, Australia, pp. 167-172, Dec. 2008.

[6] Z. Tian, D. Yuan and Q. Liang, "Energy efficiency analysis of Error Control Schemes in Wireless Sensor Networks," IEEE International Wireless Communications and Mobile Computing Conference, Crete, Greece, pp. 401-405, Aug. 2008.

[7] A. Nandi and S. Kundu, "Energy level performance of Packet Delivery Schemes in wireless sensor networks in presence of Rayleigh fading channel," International Conference on Computatioal Intelligence and Communication Networks, Bhopal, India, pp. 220-225, Nov. 2010.

[8] CC2420,http://foi.com/analog/docs/enggressdetail.tsp?fa milyld $=367 \&$ genContentId $=3573$.

[9] V. Nithya, B. Ramachandran and Vidhyacharan Bhaskar, “Energy and Error analysis of IEEE 802.15.4 Zigbee RF transceiver under various fading channels in Wireless Sensor Network", International Conference on Advanced Computing, MIT, Anna University, Chennai, India, pp. 1-5, 13-15 Dec.2012.

[10] IEEE 802.15.4 version 2006, IEEE Standards Association, http://standards.ieee.org/getieee802/download/802.15.42003.pdf

[11] Z. Zhiliang, et.al. "Performance analysis of LT codes with different degree distribution", Fifth International Workshop on Chaos Fractal Theories and Applications, IEEE 2012

[12] M. Luby, "LT Codes", Proceedings of 43rd Annual IEEE symposium on Foundations of Computer Science, pp.271-282, 16-19 November 2002.

[13] C. CongZhe, F. ZeSong, X. Ming, H. GaiShi, X ChengWen, K. JingMing, "An extended packetizationaware mapping algorithm for scalable video coding in finite-length fountain codes," Science China Information Sciences, 2013, 56(4): 042311(10). 